Fin vollstandiger oder gekurzter Abdruck der Referate ist ohne Genehmigung der Redaktion und der Verlages verboten!

\title{
Namenregister
}

Aagaard 11297. Avery 11256. Abarra 11276. A 11254.

Abbott Laboratories Awakjan 11128.

Abraham, E. $\mathbf{P}$. 11207.

Abraham, $\mathbf{S}$, 11201.

Awakjan 11128.

Babcock 11284

Beltjukowa 112

11183,11184

Bencze 11177

Bender 11223.

Babuschkina 11272. Benedict 11112.

Bacchetti 11158. Benit 11310 .

Backeberg 11112. Benkeser 11165

Ackermann 11288. Badische Anilin- \& Benson 11192.

Acqua, Dell' 11234. Soda-Fabrik Akt.- Benstz 11239.

Adalia 11227.

Adam 11259 .

Ges. 11265.11279. Benzinger 11310

Adams 11123.

Badische Bau- und Berg 11129.

A GEPHA Arbeits- Kunststof gemeinschaft zur $\quad 11276$.

Erzeugung Phar- Bailey, A. 11188 .

mazeutischer

Artikel G. m. b. H 11281 .

11250.

Baimakow 11124.

Agfa Akt.-Ges, für Baird 11122. Photofabrikation Bajez 11244. 11318. 11319. Bakelite Ltd.

Aird 11237.

Akazawa 11202. Baker 11120.

Akers 11230. Balach 11274.

Aktiebolaget Bofors Baldwin 11125 . 11250.

Balfe 11290

Akt.-Ges. für Unter- Balletto 11308. nehmungen der Balls 11204. Eisen- und Stahl- Banag 11227.

industrie 11269. Banerjee 11237.

Akulow 11137.

Alami 11245.

Albert 11284

Aldrich 11233.

Alejo 11305.

Alexander 11130

Algemeine Elektricitäts-Gesell schaft 11287

Alonso Lozano 11298

Altamirano 11194

Aluminium-Industrie Akt.-Ges. 11287

Amat 11115.

Ambanelli 11305

Ambrosio, $\mathrm{D}^{\circ}$ 11306.

Barber, E. J

11148 .

Barber, R. 11217 Barchewitz 11115 .

Bardeen 11121.

Barker 11222.

Barmore 11304

Barnett 11110.

Baronetzky 11245

Barr, F. T. 11316.

Bergman, A. G. 11139

Bergman, M. 112

Bergman, M. 112

Bergmann 11244.

Bergström 11310.

Inc. 11310

Berko 11111.

Bern 11202.

Bernhard, $\mathbf{K}$ 11232

Bernhard, S. A

11202. 11203

Bernheim 11204.

Bernstein, I. I 11239

Bernstein, M. H 11195.

Bernstein, S. 11252. Brosette 11307

Berthelot [11115]. Brossmer 11217.

Berthier 11143. Brown, B T.

Bertola 11236.

Bertsch 11135 .

Berzins 11306.

Besinger 11260

Bethell 11117

Beuss 11140

Beyer [11190].

Beyersdorfer 11

ovay 11277 .

Boyd 11169

11192 .

Boyle 11118.

rackmann 11

Bradley 11173.

Brand 11296.

Brandt 11241.

Brazier 11286.

Brazos 11113

Brennan 11181.

ritish Industria

11286.

British Petroleum

British PlasterBoard Ltd. 11276

11219

11220.

Brockemeyer 11248 .

Brody 11296

11243

Brown, J P. 11164

Brown, R. D 11143

Brückl 11308

Brunnstrom 11171.

Bryant 11136.

Bryce 11137

Bienenfeld 11209. Bublitz 11214

Barrère 11130.

Barrollier 11261.

Bietto, De 11118.

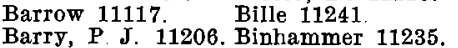

$\begin{array}{lll}\text { Barrow 11117. } & \text { Bille 11241. } & \text { Buckeley } 11254 . \\ \text { Barry, P. J. 11206. Binhammer 11235. } & \text { Buckley } 11198 .\end{array}$

Budnikow 11139 .

Ambrosioni 11206. Barsel 11251.

American Chemical Barski 11209.

Paint Co. 11289. Bartel 11256.

American Cyanamid Bartell 11129

Co. 11250. 11251. Bartholomew 11112.

11252. 11287. 11296.

Anders, E. 11113.

Anders, $H$ 11278. Baryschnikowa

Andersen 11241

Andrews jr 11233. Baudart 11109.

Andrisano 11170. Bauer 11267

Andrus 11228.

Anguera 11215 .

Anissimow 11135 .

Anner 11253

Anson 11193.

Antes 11120 .

Anttonen 11243.

Appareils et Eva-

porateurs Kestner 11266 .

Arcos, J. C. 11197.

Arcos, M. 11197.

Arens 11317

Armstrong 11226

Arrigo 11223 11234.

Arrott 11123.

Arthur 11256

Asaro, D' 11129.

Ashbrook 11280.

Askonas 11232.

Asplund 11310 .

Asselineau 11208.

Astrup 11244.

Atwood 11171 .
Bird 11293

1118. 1111.

Bartlema 11248.

Baryschnikow

11137 11150

Basolo 11136.

Birks 11129.

Bitler 11207.

Black 11316.

Blaha 11131.

Blair 11112.

Blamont 11115.

Blau [11296]

Block 11135 .

Blockstein 11109

Bobeth 11314.

Bode 11304.

Böhner 11279

Böhringer 11216 .

Böltz 11259.

Baum 11212. 1276 . Börner 11300.

Büddicker-Löns

11294

Bühler, Gebr.

11265

Buffa 11305.

Buffet 11268

Bujewskoi 11309.

Bunge [11264]

Bunk 11285

Buogo [11308].

Burch 11118

Burg, A. B. 11150 .

Burg, W J van der

$$
11179
$$

Burgoyne 11148

Burkart 11298.

Baumann, N. P

Böttcher, A 11276. Burley 11311.

11269. Bogan 11130.

Bohon 11273.

Boikow 11160 .

Boivin 11255 .

Bollow 11157.

Bone 11221.

Butcher 11173.

Butler 11112 .

Butt 11262

Buu-Hoi 11197.

Buzi 11308.

Beck 11263

Byrne 11200.

Becker, R. A. 11237 11297

Boorse 11126.

adier-Eberhard

Becker, R. L. 11112.

Bedo 11116.

Beer 11173.

Beiler 11243.

Beiser 11313.

Bell Telephone Laboratories 11268.
Ltd. 11251. Co. California Res. 11308 .

Borchert 11141.

Bornstein 11118.

Bosch, R.

$$
11268 \text {. }
$$

lingaert 11148

Calvet 11138

Camand 11209

Campbell, F. W. 11235.

Bottenbruch 11261. Campbell, P. N. 11232.
Burckhardt 11186.

Böttcher, G. 11124. Burnside 11289.

Bvatt 11110.

Bell, W. C. 11284 . Inc 11266 11267. Boschi 11201.

Cady 11148 .

Canonica 11158.

Capuano 11305.

Carassiti 11135

Carman 11278.

Carminati 11237.

Carrick 11297.

Carrière 11289.

Carter 11207

Carver 11115.

Case, J. D. 11233. Corrigan 11264. 
Doletal 11:54

Dombrowskaja 1112

Dominé-Bergès 11139

Dominitz 11167.

Donahoe 11176

Dorfman 11232

Dorman Long

Ltd. 11299

Dormer 11315.

Dornow 11228.

Dorr Co. $11286^{\circ}$ 11287

Doucet 11123

Doud 11218.

Doudoroff 11199

Dougall 11207.

Douglas, B. I 1135 .

Douglas, R. A. 11119

Douglas, W. W 11235 .

Dow Chemical Co. 11254. 11279

11309

Downey 11.226 .

Euler, \& 1122

Gatiney 1125

Heng $D 11224$ Gaiud 11170

Evans, J S. 11110. Gaizauskas 11117

Eyring 11119

Fabre 11272

Falk 11125 Gale 1123 Gambussi 11234

Fallentin 11244 Gantz 11147

$\begin{array}{ll}\text { Farbenfabriken } & \text { Gapuz 11227 } \\ \text { Gardiner 1125 }\end{array}$

Bayer Akt.-Ges. Gardner 11113. 11295

Garkavi 11260

Farbwerke Hoechst Garlick 11222 Akt-Ges, vor- Girman 11277 mals Meister Lu- Grarnatz 11305. cilus \& Brüning Garola 11.277 11253 11292. Gaspari 11201 11295 Gauthice 1 [260.

Farnsworth 11121. Geballe 11118.

Farrow 11210. Goer 1131

Farwell 11112. Gegusin 11131.

Fauconnier [11110.] Géher 11244

Fechner 11280 Geldmacher-

fedorisehtschewa Hillinckrodt

11309 11261.

Fed rtschuk 11140. Geller, I. A. 11277

Iredotow 11133. Geller, Z. 11118.

Drake C. F. 11269 Feichtinger 11299. Gollért 11186

Drake, L. R. 11279 Feinberg 11239. General Aniline

Dranen, van 11126. Fenessy 11314. Film Cory.

Draper 11278. Fenske 11316.

Drescher-Kaden Ferguson, A.J. 11124

11268 . 11295

11319

11112.

Dreyfus 11319

Drickamer 11145.

Drier 11141

Droeven 11278

Drubljanetz 11271.

Dshelepow 1111

Dshemuchadse 11212.

Ferguson, B C. 11220 .

Ferguson, M. H. 11226 .

General Dry Batteries, Inc. 11269

General Electric Co. 1126711270 11302 .

ernclius 11135 .

Fernholz [11190].* Gensler 11189

Ternseh G. m. b. H. Georgi 11308.

11288.

Gerard 11235.

Gerbaux 11192

Gerber 11154.

Gerlieim 11225

Gerlich 11239

Germann 11251

Germer 11118

Gerrard 11155.

11161 .

Dunn, G. H. 11118. Fineberg 1122

Dunn, M. S. 11229. Finerty 11235

Du Pont de Nemours Fink, K. 11199

E. I , \& Co. 11300 . Fink, R. M. 11199. 11313.

Dušinsky 11249 .

Duval 11138.

Dwarakanath 11212 .

Earp 11298 Finney 11305

Gerstner 11244

Gertsman 11281

Gesellschaft fü

Eiscella 11296 .

Fisch 11299 .

Industrie in 11295

Fisher, L. H 11118. Gesellschaft für Fisher, W. E. 11313. Elektrometal-

Eastman Kodak Co. Fishman 11145

11290.11291.

11313. 11318.

Eckert 11221.

Edwards 11238.

Egelhaaf 11194.

Egle 11213

Fodor 11174.

Ehrhart, $H$. 11253. Food Machinery

Eibl 11200.

Eichinger 11211.

Eiduss 11154 .

Eigen 11274.

Eilersen 11244.

Eiseman 11225.

Eiseman 11225 .

Eisenhuth 11313.

Elektrokemisk A/S 11272

Frazier 11241.

Flrick 11223

Eirick 11223.

Enderlin 11294.

Ennis 11284.

Erbel 11279 .

Erbring [11129].

Ercoli 11237.

Eriksen 11282

Friksson 11307.

Ernsting 11250 .

Esch 11280.

Esso Research \& Engineering $\mathrm{Co}$. 11297.11316 . 11317.
Fitelson 11307

Fleischhacker 11251.

Fleury 11155

Flörke 11273.

Floyd 11225 . Chemical Corp. 11293.

Fordham 11191 Forosstjan 11159. Forst 11146.

Trortenbaugh 1115

Fougere 11119

Fox, J. E. 11292.

Fox, R. E. 11120.

Fraenkel 11147.

Freard [11279]. 11287

Gevaert Photo Producten N. V. 11320.

Giancola 11252

Gibbons 11117 .

Gibian 11261.

11182.

Gilbert 11212

Gilleo 11123.

Gillmor 11313.

Gilman 11168.

nsberg 11283

Gisvold 11293.

laduschtschenko

11139

Glascock 11234

Glawitsch 11124

Trederickson 11204. Glazunow 11316.

Frey 11122.

Fried 11315

Fröhlich 11122

Frölich 11307

Fromageot 11218.

Frost 11244.

Fuchs 11245.

Fukano 11131.

Fukuba 11303 .

Fukuto 11236.

Fuller 11266.

Funnell 11240.

F'uson 11145.
Gleason 1129

Glon 11217

Gluckstern 1111:

Godin 11232

Godlove 11115 .

Goering 11152

Götzberger 11131

Goffart 11221.

Gohr 11316

Gold 11121

Goldberg 11292.
Goldman 11123.

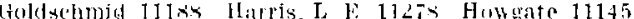

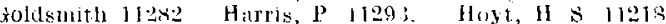
Goldstein 11145 Hart, J' (' 11221 Hoyt, R. S 11218 Goldswotthy 11237 Hart, R W 11115. Huber, J M, Corp. Golowanow $11309011116 \quad 11297$

Golown 11112 Harin 11120 Hubert 11300

Gomez 11305 Hartford National Hüttig 11124

Gomori 1120\%. Bank \& 'Trust co. Hughes, E. C Good 11113 11297 11265.

Goodman, J J. Hattori 11213. Hughes, R. C.

11207. Haul $11136 \quad 11270$

Goodman, J W. Hauser 11168 Hugus jr 11140.

Haxo 11211

Gorbatschew 11150 Hayaishi 11205

Gorodysski 11196 Hayes 11299

Goronowski 11271 Haynes 11267

Hedberg 11282 .

Hedges 112 S6.

Heicle 11312.

Gottlicb 11207

Gould 11260

Gouzène 11311 .

Gove 11112

raff-Baker 11291. Helweil 11196. Huttl 11284

Grafstein 11160. Hein 11180

Graham 11286

Heite 11237

Grant 11.217

Illari 11159 .

Grassner 11265. Hellauer 11236.

Gray, E, O 11115. Hendershot 11236. 11116

Henderson $j r$.

aly, G. W. 1114911151.

Gray, J J 11287 Henliel, F. 11275.

Grevo 11246 Henkel \& Cie

$\begin{array}{ll}\text { Green, A. A. } 11205 & \mathrm{G} \text { m b. H } \\ \text { Green, A. E. S. } & 11290 \text {. }\end{array}$

11110 Hemnicke 11279.

Henson 11305 .

Greenwood 11278. Hepler 11140.

Gregson 11280.

Grenior-Besson 11115

Giiboff 11195

Griehl 11311.

Griffith 11229 .

Giilly 11255 .

Grlic 11237

Grobecker 11283.

Grodsky 11261.

Gross, D 11206.

Gross, F. 11219.

Grissman 11220

Grosvalet 11119

Groth 11291

1115

Hepler 11140.

Hercules Powder Co. $11267 \quad 11279$

Hermon 11257

Herold 11190.

Herout 11213.

Herr 11234

Herring 11119

Hershberger 11243.

Hertlein 11226

Heseltine 11318. 
Johnson, C $\mathrm{E}$
$11 \mathrm{~L} 27$

Jolinson, G. B.

$11 \div 71$

Johisson, W $B$. 11289

Johnston 11209

Johnstone $11 \geq 72$

Jones $A$. 111265. Kortim [11127].

Joues, B. 11149. Kostk 11232

Jones, W. D. 11373 . Kosola poff 11182.

Jones, W. W 11215. Kozdrojowna 11179. Lewis, Y. S. 11212. Maniwa 11 132.

Jordan jr. 11225. Krantz 11222. Licentia Patent-

Joshi 11231 .

Josien 11145.

Joyee 11212 .

Jud 11298 .

Jülicher 11295.

Juroff 11233 .

Jursich 11151.

Juza 11123.

Kalimann 11146.

Kanda 11248.

Kane 11191.

Kaplan 11115.

Kari 11213.

Karjakin 11153 .

karsten [11254].

Karten 11153

Kasai 11238.

Kasakowa 11271.

Kistel 11248 .

Kato 11113

Katz, J. 11201.

Katz, L. 11115.

Jiatz, M. 1.235 .

Katzon 11110 . Kutzim 12239

Kaufmann 11296. Kwak 11145 .

Kautsky 11214.

Ke 11133

Keller 11276 .

Kelley 11229

Kellogg, M W.,

Co. $11289 \quad 11317$

Kemball 11153

henip 11121.

Kenaga 11279 .

Kendill 11111 .

Kerlee 11112 .

Kern $11190 \quad 1$

Fessler 11248 .

Ketschek 11263

Ketschek 11263

Keyes 11121 .

Kicssling 11287

Kikutani 11148 .

Kikuth 11245 .

Kranzler 11246.

m. b. $\mathrm{H} \quad 11267$.

ratz 11188

Kratipp $112+0$.

hrebs 11290.

Kretsclumer 11303. 11124.

Lind 11243

Kritehevsky 11228. Linderström-Lang

Krone 11112

Krystak 11.165 .

Kubelka 11271.

11217.

Kulski 1127:

Kumar 11214.

Kuninsky 11281 .

huo 11282

Kurath 11111.

kury 11140

Liesegang 11313.

Light 1126 I

Lina-de-liaria

11.193.

Linisay 11168.

ling 11200.

Linker 11216.

linnig 11:254.

Lippe 11310.

Jissol 11310

Litherland 11112

ljuiwinski 11274.

Jjung gren 1.1231 .

lochner 11242.

Jucke 11280 .

wokheed Aircraft

Corp 11300.

löfgron 11222 .

Juges 11249 .

Lohr 11279.

Laberriorue-lirolow 11113

Laboratuires Tran-

cais de Chimio-

therapie 11253

Lacassagne 11197

Lacombe 11:16.

Litcroix [11198]

Lal $1130^{\circ}$.

Landija 11126.

Iondon 11225.

Jong 11296.

Longden 11281.

Lominac 11284

Lorenzen 11315.

Josinski 11133.

Low 11118.

Lowick 11206.

Loxton 11219.

Lucas 11233.

Ludlow 11300.

Lang 11315 .

Ludwig 11210.

Langenbeck 11157, Iü̈cke 11285.

Lape 11240.

Irapis 11198.

Lappert 11155.

Laquet 11.299

Lardy 11200 .

Lnener 11201.

Lilrsen 11152.

Kimpton 11273

King, I. R. 11119. Larson 11302.

King, N. 11306.

Kiperman 11122 .

Kipping 11298.

Kijprianow 11175

Kircheis 11217 .

Kirk 11250.

Kiwman 11260

Klarmann, E. G. $11: 50$.

Klarmain, $\mathrm{H}$ $11: 67$

Klaus 11147 .

Kleber 11303

Laschko 11128.

Lasman 11303

Lasnitzki 11196 .

Lassieur 11258.

Latimer 11140.

Latour 11313.

Laubenheimer

11274

Laurie 11146 .

Lawendel 11163.

Lawson 11145.

Lax 11121.

Lazarus 11112.

Kleinwächter 11288. Leachman 11113.

Klemens 11121. Leaf 11216.

Kling 11265

Lecomte 11138.

Klitward 11222. Le Corre 11120.

Knoll Akt-Ges., Lederer 11208

Chemische Fabri- Lee, A. 11229.

ken 11251 .

Kobinger 11240

Koch 11293.

Kugl 11233.

Kohler, R. 11290. Lehmann 11245.

$\begin{array}{ll}\text { Köbler, U. } 11234 . & \text { Leitz, E., G. m. b. } \\ \text { Köller, V. 11219. } & \text { H. } 11275\end{array}$

Köhne 11274.

Koelle 11203 .

Köster 11310

H. 11275

Lenger 11277 .

Lepore 11111

Kolıler, K. 11141. Les Fils de Victor

Kohler, W 11310 . Michel (Soc a.

Kojima 11230.

r. 1) 11319 .

Lessin 11241.
Lüdecke 11245. 11239.

Lurgi Gesellschaft für Würmetech-

nik m. b. H. 11290 .

MeCall 11164

MeCandless 11228.

Mcclellan 11313.

MeColium 11214.

McConkey 11217.

MeConnel 11178.

McCormick 11207 .

MeCullough 11272.

MacDonald 11111.

McElroy 11205.

Macliarland 11269 . 11117.

Mefarlane, S. B. 11313.

11199. Mett

MeGlory 11242 .

McGuire 11123.

Machiki 11207

McIlwain 11203.

Mackler 11229.

MeLean 11266.

MoNickle 11201

Madsen, L. L. 11278.

Madsen, V. M

11118.

Märkische Seifen-

Industrie 11302

Magat 11298.
Lützenkirchen

Lukjanow 11112.

McGrath 11317.

Magri 11287

Mlaher 11305.

aler-Bode 11298

Maier-Hüser 11218

Malaytesta 1.180.

Malkowa 11137

Landel, J 11254.

Mandel, Z, 11166.

Mann, A. 11125 .

Manning 11284.

Marburiger 11120.

March 11236

Marcot $11: 87$.

Margrave 11127

Haris 11112

Markham 11263.

Martín, $\mathrm{D}, \mathrm{T}$.

$11: 282$

Martin, G. J. 11243.

Martin, H. C. 11114

Martin, L. 11277.

Martinson 11283.

Martinsson 11243

Martius 11140 .

Martynoff 21143.

Marullo $1 \mathrm{~J} 195$.

Marzin 11307.

Masing 11285.

Masoni 11205.

Mathes 11293.

Mathews 11232.

Mathieu 11227.

Matbys 11265 .

Matsen 11149.

Matthews 11195 .

Maude 11292.

Maurin 11209.

Malvroides 11121.

ax-Planck-Ge-

Förderung der

Wissenschasten

e. V. 11269

Maximenko 11315

Maxon 11236

ray, R.-M. 11221. ay \& Baker Ltd. 11291.

Mayer 11172 .

Mazia 11195.

Meadow 11181.

Medvedieff 11268.

Meeker 11136.

Meerman 11314.

Meggers 11116.

Meier, J. W. 112

Meinhart 11282 .

Meinke 11114

Meinwald 11143 .

Melichar 11180

Melnikow 11269.

Melvin 11111.

11268.

Metcalf 11236.

Metrikina 11168.

Ietropolitan-Vic-

Co. Ltd. 11268.

11289.

Metzger 11308. 

1122

Patterson, H. R.

11157

Patterson, $J$ W. 11223.

Paukner 11303

Paul 11112

Pauling 11193.

Payens 11128 .

Payot 11169 .

Peard 11303.

Pearson, D. E. 11154.

Pearson, R. G. 11136

Peaslee 11110

Pederson 11115 .

Peet 11303 .

Peet 11303 .

Peiler 11167.

Pekkarinen 11221.

Pelletier 11186.

Peltola 11221.

Pénau 11253.

Perceau 11302.

Perkins 11112.

Perko 11144.

Perry 11219.

Pers 11234.

Pérszász 11239.

Peteri 1131

Peters, K. 11198.

Peters, T. 11262

Peters, W. 1126

Peterson 11120

Petroff 11311.

Petrovicki 11265.

Petrow, A. A.

11156.

Petrow, A. D. 11160

Petrow, V. 11178.

Petseh 11228.

Petty 11207

Petzold 11275.

Phelps 11207.

Philips Electrical

Industries Ltd. 11270.

Phillips Petroleum Co. 11260.

Philpot 11195.

Pickard 11256.

Pickels 11255.

Pickering 11285 .

Pietsch 11290 .

Pillai 11305 .

Pillat 11236.

Pines 11121.

Pino 11227.

Pippard 11iz2.

Piroux 11270.

Pisani 11234.

Plazek 11179

Plongeron 11218.

Pochapsky 11288.

Poetter 11282.

Polubojarinow

11274.

Ponomarenko 1

Ponzoni 11206.

Poole 11252

Pope 11154

Popilski 11274.

Popjak 11204.

Popow 11127.

Porai-Koschitz 11174.

Poroschin 11260.

Porter, F. W. B. 11316.

Porter, H. T. 11316.

Porter, J. W. 11212.

Porter, L. K. 11215 .

Portnjagina 11175.

Portnow 11258.

Post 11129

Postgate 11198.

Pott 11266.

Pouradier 11140.
Power 11110.

Prem 11301.

Plener 11270

Preston 11241.

Prévot-Bernas

11298.

Prieur 11280.

Prigogine 11125. 11136.

Prince 11134.

Prosser jr 11112

Pugatsehewitsch 11181.

11128. Robinson, F.A.

Pugh 11123 11228

Pumerer [11190].* Roche 11216.

uackenbu $\mathrm{h}$ 11212 .

Quelet [11190].

Querner 11217.

Raab 11279

Rabourn 11212

Rader 11279.

Radio Corp. of America 11270.

Raffel 11128.

Ralston 11267.

Ramakrishna 11147.

Ramakrishnan 11205.

Ramart-Lucas

11143

Ramsay 11117.

Rao, K. S. 11130 .

Rao, P. N. 11170 .

Rapoport 11225

Rapport 11216

G I. H. 11293. Rudolph 11307.

Rasmussen 11111. Rühl 11285.

Ravenna 11164

11203.

Record 11208.

Reeves $11110 . \quad$ Rushton 11235.

Regie Industrielle Rustigian 11209 de la Cellulose Rutledge 11120 .

Coloniale 11311. Ruyven, van

Reichle 11265 .

Reid 11157

$$
11137
$$

Rybtschinski

11271.

Reilly H. O. 11198. Rychlewska 111

Reliephotographie, Saarinen 11228

La, Soc. pour Sacharova 11155

l'Exploitation des Sadler, F. S.

procédés de photo- $\mathbf{1 1 3 0 8}$

graphieenrelief Sadler, P. W. M. Bonnet 11319 .

Reilly, W. L. 11171.

Reindel 11209

Reinhart 11147 .

Reinisel 11298

Reinius 11234.

Reiser 11185 .

Reiter 11242.

Rekker 11250 .

Relyea 11152 .

Renker-Belipa

G m. b. H. 11

Research

11251.

Rothwisch 1128

Reuss 11125.

Reynolds 11120 .

Rexroad 11145 .

Rhoads 11198 .

Richert 11198.

Richter. A. 11276.

Richter, L. 11295.

Riddell jr. 11125 .

Rider 11147

Rieche [11293].

1129711298.

Riehl 11118.

Riemenschneider

11224.

Riester 11318.

Riezler 11281 .

Salathiel 11315
11143.

Sagel 11285.

Sagjanski 11140.

Sahay 11214.

Sahni 11115 .

Saint Gast, de 11316.

Saitzewa, A. F.

11309.

Saitzewa, J. D. 11142 .

Sakamoto 11260

Sakellaridis 1111

Samitels 11302.

Sandréa 11141.

Santini jr. 11307.

Santor 11244.

Saremba 11277

Sasaki 11214.

Sato 11205.

Sattelberg 11218

Sallermilch 11293 .

Sauvage 11250

Savagnone 11219

11224.

Savidge 11219.

11220.

Schabarowa 11142. Scliwenker 11.292.

Schachman 11255. Scoffom 11286.

Schaefer 11288. Scott 11212.

Schafer 11257 .

Schulten 11114.

11275.

chultheig, $P$

11270

Schultheis \& Söhuo

11275

Schafmer 11207 Sebban 11298

schaidurow 11310. Seckel 11299.

seel 13137 .

Schamschew 11112. Seher 11248.

Schanker 11198 Seifert 11264.

Scharkow 11309 Sekiya 11248.

Schauenstein Seligman 11203

Selinskaja 11273 .

Semple 11226

Sen Sarma 11113.

Setäla, H. 11197.

Setälä, K. 11197

Seubold jr. 11145

$\begin{array}{ll}\text { Scheindlin } 11229 & \text { Seubold jr. } 11 \\ \text { Scheirer jr 11254 } & \text { Shafei 11243. }\end{array}$

Schenck, G 11245 Shapiro 11298.

Scherhag 11190. Sharples Corp.

Schibler 11294 \$1308.

Schikanowa 11294 Shay 11197

Schild 11261. Sheehan 11286.

Shepherd 11161.

Sheppard 11117.

Shernowskaja

11155.

Shilts 11125

Shimokoriyama 11213.

Schlenker 11300.

chleyer 11194.

.

Sclimeckenbecher

11123

Schmid 11177

11191

Schmidt Karl-

Heinz 11253.

Schmidt, P. 11123 .

Schneider $\mathbf{H}$.

11235.

Schneider, W.

11318.

Schneidewind

11281.

chnetzer 11306.

11289 .

11289.

Schoep 11141.

Scholer 11244.

Schpanow 11186.

11208.

11209

Schreiber 11241.

Schreier 11218.

Schueler 11210.

Schürmeyer 11242 .

Schulman 11110. 


\begin{tabular}{|c|c|c|c|c|c|}
\hline $\begin{array}{l}\text { Arinska Skifterolje } \\
\text { Aktiebolatget } \\
11315\end{array}$ & $\begin{array}{l}\text { Thorn Electrical } \\
\text { Industries Ltal. } \\
11270 .\end{array}$ & $\begin{array}{l}\text { Jmeda } 17148 \\
\text { Umhoefer } 11293 . \\
\text { Ungemach } 11275 .\end{array}$ & $\begin{array}{l}\text { Vitamins Itd. } \\
\text { 1las:2. } \\
\text { Vlugter } 11289 .\end{array}$ & $\begin{array}{l}\text { Wendlandt } 11136 \\
\text { Wendt } 11272 \\
\text { Werner, G. } 11240\end{array}$ & $\begin{array}{l}\text { Worley } 11126 \text {. } \\
\text { Wrba } 11217\end{array}$ \\
\hline Swayne $112+3$ & Thorne 11:15. & United States of & Vogel 11273. & $r, H . W$. & Wright, D A. \\
\hline swen & Thorsen 11111. & America, Secre- & Vogt, if 11220. & 11210 & 18. \\
\hline Swerdlow 11129 & Thye $112 \pm 4$ & of the Army & Vogt, W 11224 & Werz 11211. & Wright, E. S. \\
\hline (i11 11169 & Ticboes 11301 & & Voigt 11278 & selaja 11196. & 250. \\
\hline ecki 1 & Tiefontlal 11171. & d States Ato- & Wacha 11259 & 11220 & Wright, G. F'. \\
\hline Sy, & Tiety l120.1 & gy Com- & ranejewa & Westall 11227 & 87 \\
\hline Sziman 11317 & Tiggelman-v & mission 11287. & 11142 & Westerfeld 11198. & Wright, J. G. E. \\
\hline Szu & ten 11208 . & 1 states Rub- & Wachruschew & inghouse & 01. \\
\hline Tab & Timan 1112 & 1299 & 11 & orp. 11 & Wris \\
\hline 64 & Timofejewitschewa & Upjo & 11209 & ey 11181 & \\
\hline Tachikawa & 11128 & 33 & Wadey 11115 . & White, G K $\mathbf{1} 1121$. & che 11244 \\
\hline Tace & Titow 11150 . & 11164. & h 11119 & White jr., J W & erly 11216. \\
\hline Tiaha 11209 & 'Tkatschenko 1 & 11109 & Wagner, H 11232. & 05 . & 7. \\
\hline Takacri & Torne, $\mathrm{v}$ I & $\mathrm{i}, \mathrm{F} 11202$ & Wagner, J 113 & nack 11147. & Ia 111 \\
\hline 'lak & Tokunata 1 & i, M. 11202 . & Wagner, R. 11 & er 1131 & uchi, s \\
\hline $\mathrm{Tal}$ & & 11122. & aftig 111 & ham 11317. & \\
\hline 'Ialmage 11 & Tolm & 56 & a 11210 . & Wiebe, J. 11265. & uchi, T. \\
\hline in 11 & Ton & $\mathrm{Vi}$ & on 1 & c, S 11265. & \\
\hline $\mathrm{Ta}$ & ulian & 294. & г 11 & nack 111 & Ya \\
\hline T:L & ey 112 & & e 11 & $1126 \mathrm{~b}$. & $\mathrm{Y}$ \\
\hline Til & tta 11 & nt:sches & I 11255 . & s 11298 & hara 11260 . \\
\hline $\begin{array}{l}\text { Tarver } 11206 . \\
11261\end{array}$ & $\begin{array}{l}11288 . \\
11233\end{array}$ & Rod- & $\begin{array}{l}\mathrm{n}_{\mathrm{S}} 11217 \\
\mathrm{~S} \mathrm{Y}\end{array}$ & ur 11204 & D. \\
\hline k 11114. & no 1122 & nfabrik & Y N. N. & $\mathrm{ns}, \mathrm{C} . \mathrm{M}$. & $\mathbf{Y}$ \\
\hline 'Ta & $\operatorname{man} 1$ & 1297. & & & $\bar{Y}$ \\
\hline er 11219 . & Tredgold 11110 . & 11298.11301. & nk 11125 . & Williams, D. C. & is 11296 . \\
\hline & $\begin{array}{l}\text { Triggs } 1131 \\
\text { Tristram } 11\end{array}$ & $\begin{array}{r}11314.1131 \\
\text { VEB Kombin }\end{array}$ & $\begin{array}{ll}\operatorname{ck} & 1 \\
\mathrm{rg} & 11\end{array}$ & 62. & \\
\hline $\begin{array}{l}\text { Taylor, D. A. H. } \\
11237\end{array}$ & $\begin{array}{l}\text { Troschin } 111 \\
\text { Trott v 11: }\end{array}$ & wohl" & $\begin{array}{l}\text { n } 11300 . \\
11274\end{array}$ & $\begin{array}{r}1 \\
\text { Wil }\end{array}$ & \\
\hline Taylor, G M. & Trotter 1123 & VEB & Wawzonek 112 & son 11 & $\mathrm{Zs}$ \\
\hline 11 & $\operatorname{Tr}$ & & We & 1118 & 38. \\
\hline Tem & Tsao 11140 & an der & Weber, H. 11 & pot Corp. & 78 \\
\hline Te & T's & &, $\mathrm{U}$ & & $\mathbf{Z e}$ \\
\hline $\mathrm{Te}$ & $\mathrm{Tsc}$ & $\mathrm{Ve}$ & & $\mathbf{Y}$ & $\mathrm{Ze}$ \\
\hline $\mathrm{Tc}$ & 11206 & 111 & n 11 & er 11118 . & 10. \\
\hline 'Th & Tuft 11244 & nan & We & hage & \\
\hline Th & gin 113 & & alh & I 1 & \\
\hline Th & nes 11 & ate Alu & & De 11 & $\mathrm{Zie}$ \\
\hline & Tu & 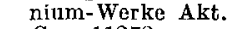 & We & 5. & \\
\hline & $\mathrm{ch} 11$ & 11 & nd 11 & mirskaj & \\
\hline 11300 & Turkewitseh & igte Kork- & & & Zillig 112 \\
\hline $\begin{array}{l}\text { Thomipson, C. } 2 . \\
11210 .\end{array}$ & ni $[1]$ & $\begin{array}{l}\text { istrie Ak } \\
11276 .\end{array}$ & $\begin{array}{l}\text { Weiss, C F }[1 \\
\text { WeiB }_{\mathrm{K}} 111\end{array}$ & Woj & ka 112 \\
\hline Thor & 11181. & 11109 & enber & Wo & Zschimmer \& \\
\hline & & & & & \\
\hline $\begin{array}{l}\text { Thompson, } \mathrm{S} \\
\text { [11110]. }\end{array}$ & $\begin{array}{l}11234 . \\
\text { ohde } 11\end{array}$ & Vijayaraghavan & $\begin{array}{r}1 \\
\text { Wei }\end{array}$ & $\begin{array}{l}\text { Woods, J. } 1 \\
\text { Wood, W } \mathbf{H}\end{array}$ & 308. \\
\hline 112 & Uhlenbeck 1112 & Villi & 11 & bury 11 & $\mathrm{Zu}$ \\
\hline 112 & Ulden, van 1 & anen 11 & Welch 11117 & dward & \\
\hline
\end{tabular}

\section{REINHARD KRZIKALLA}

\section{Rechentafeln zur chemischen Elementar-Analyse}

1956. 232 Seiten. Kunststoffeinband DM 12, -

„... Man kann mit diesen Rechentafeln zwei Rechenarten sicher und schnell ausführen: Die Berechnung der prozentualen Elementarzusammensetzung einer chemischen Verbindung sowie das Aufstellen einer Bruttoformel aus gefundenen Prozentzahlen. Die kurze Anleitung nebst Vorwort gestattet sehr schnell die Einarbeitung in den Gebrauch der Tabellen, mit denen man Verbindungen bis zum Molekulargewicht 1000, enthaltend die wichtigsten Atomarten, Metalle wie Nichtmetalle, berechnen kann ....

Die Pharmazeutische Industrie

Prospekt auf Wunsch

VERLAG CHEMIE - GMBH - WEINHEIM/BERGSTR. 